\title{
Prediction of 8-state protein secondary structures by a novel deep learning architecture
}

\author{
Buzhong Zhang ${ }^{1,2}$, Jinyan $\mathrm{Li}^{3}$ and Qiang Lü ${ }^{*}$
}

\begin{abstract}
Background: Protein secondary structure can be regarded as an information bridge that links the primary sequence and tertiary structure. Accurate 8-state secondary structure prediction can significantly give more precise and high resolution on structure-based properties analysis.

Results: We present a novel deep learning architecture which exploits an integrative synergy of prediction by a convolutional neural network, residual network, and bidirectional recurrent neural network to improve the performance of protein secondary structure prediction. A local block comprised of convolutional filters and original input is designed for capturing local sequence features. The subsequent bidirectional recurrent neural network consisting of gated recurrent units can capture global context features. Furthermore, the residual network can improve the information flow between the hidden layers and the cascaded recurrent neural network. Our proposed deep network achieved $71.4 \%$ accuracy on the benchmark CB513 dataset for the 8-state prediction; and the ensemble learning by our model achieved $74 \%$ accuracy. Our model generalization capability is also evaluated on other three independent datasets CASP10, CASP11 and CASP12 for both 8- and 3-state prediction. These prediction performances are superior to the state-of-the-art methods.
\end{abstract}

Conclusion: Our experiment demonstrates that it is a valuable method for predicting protein secondary structure, and capturing local and global features concurrently is very useful in deep learning.

Keywords: Protein secondary structures, Q8 prediction, Local block, Deep learning

\section{Background}

A protein is a linear chain of amino acids connected by peptide bonds. The primary structure of a protein is just the amino acid sequence ordered in the polypeptide chain. Repeated regular conformations on the polypeptide chain are called the secondary structures of proteins. From the secondary structures, a protein can be folded into a stable three-dimensional structure, which is called the tertiary structure of a protein. Although a protein's structure is largely determined by its amino acid sequence [1], advanced studies show that accurate prediction of tertiary structures from sequences is a challenging problem currently with poor performance. The prediction of protein

\footnotetext{
${ }^{*}$ Correspondence: qiang@suda.edu.cn

'School of Computer Science and Technology, Soochow University, Suzhou, China

Full list of author information is available at the end of the article
}

secondary structures from sequences is then considered as an intermediate problem bridging the gap between the primary sequences and tertiary structure prediction.

Protein secondary structures are traditionally characterized as 3 general states: helix $(H)$, strand $(E)$, and coil (C). From these general three states, the DSSP program [2] proposed a finer characterization of the secondary structures by extending the three states into eight states: $3_{10}$ helix (G), $\alpha$-helix (H), $\pi$-helix (I), $\beta$-stand (E), bridge $(B)$, turn $(\mathrm{T})$, bend $(\mathrm{S})$, and others $(\mathrm{C})$. Prediction of the three states from protein sequences (i.e., the Q3 prediction problem) has been intensively investigated for decades using many machine learning methods, including the probability graph models [3, 4], support vector machines [5, 6], hidden Markov models [7, 8], artificial neural network [9-12], and bidirectional recurrent neural network(BRNN) [13-16].

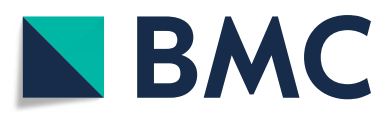

(c) The Author(s). 2018 Open Access This article is distributed under the terms of the Creative Commons Attribution 4.0 International License (http://creativecommons.org/licenses/by/4.0/), which permits unrestricted use, distribution, and reproduction in any medium, provided you give appropriate credit to the original author(s) and the source, provide a link to the Creative Commons license, and indicate if changes were made. The Creative Commons Public Domain Dedication waiver (http://creativecommons.org/publicdomain/zero/1.0/) applies to the data made available in this article, unless otherwise stated. 
Recently, the focus of secondary structure prediction has been shifted from Q3 prediction to the prediction of 8 -state secondary structures, due to the fact that a chain of 8-state secondary structures contains more precise structural information for a variety of applications. The prediction of the 8 states of secondary structures from protein sequences is called a Q8 prediction problem. The Q8 problem is much more complicated than the Q3 problem. Because it is considerably more complicated than Q3 prediction, deep learning methods have been applied. For example, SC-GSN network [17], the bidirectional long short-term memory (BLSTM) method $[18,19]$, the deep conditional neural field [20], DCRNN [21], the next-step conditioned deep convolutional neural network $(\mathrm{CNN})$ [22] and Deep inception-insideinception (Deep3I) network [23] have been widely explored.

Protein secondary structures are not confined to only adjacent residues, but also involved with long-range residue contacts. Many literature computational methods have considered these biological facts to combine both local and long-range contact information. DeepCNF [20] is a Deep Learning extension of Conditional Neural Fields, which combines the advantages of both conditional neural fields and deep convolutional neural networks. DCRNN [21], comprised of a multi-scale convolutional layer linked by three stacked bidirectional recurrent network layers, uses $\mathrm{CNN}$ to obtain the local information and BRNN to obtain long-range contact information. An ensemble of ten independently trained DCRNN has achieved a 69.7\% accuracy on the CB513 benchmark data set. Next-Step Conditioned CNN [22] combines the previous labels to the current input to remember the former information like RNN. It further improves the prediction performance to a $70.3 \%$ accuracy. When trained under an ensemble learning framework, it has achieved a 71.4\% accuracy, representing the newest state-of-the-art performance of the Q8 prediction problem. Based on the Google Inception network [24], a Deep inception-inside-inception (Deep3I) network [23], named MUFOLD-SS which are mainly constructed by CNNs and residual networks(Resnet) [25], is proposed. MUFOLD-SS uses inception-inside-inception and Resnet to enhance the performance of capturing longrange contact information in sequences. MUFOLD-SS has been evaluated for the Q8 and Q3 prediction performance on the CB513, CASP10, CASP11 and CASP12 datasets. Very recently, Port 5 [16] assembling seven BRNNs have achieved $73 \%$ and $84.2 \%$ of Q8 and Q3 prediction on 3315 protein sequences respectively.

In this study, we propose to use a convolutional, residual, and recurrent neural network (CRRNN) for both Q8 and Q3 secondary structure prediction. Firstly a local block comprising of one-dimensional CNNs and the original input combines local features and original sequence information. After local block filtering, the sequences are fed to a bidirectional recurrent neural network (BRNN) containing gated recurrent units (GRU) [26]. This architecture of BRNN can model the sequence structure and can capture long-range dependencies of the residues. The BRNN is a three-layer stacked structure with residual connections [25] linked to the interval BRNN layer. To reduce the high-dimensionality of hidden-layer input, a 1D convolutional filter with one kernel [24] is used along with the residual connection. The multi-perception and softmax layer for the final classification are then connected. We used 12,148 sequences to train the model and tested its performance on the benchmark data sets CB513, CASP10, CASP11 and CASP12. We also trained ten individual model and ensemble them as a integrated model named as eCRRNN. The prediction results have demonstrated that the deep network has better generalization performance in comparison with the best existing method. The superior performance is mainly attributed to: (i) The local block can integrate both local features and the original sequence information; the $1 \mathrm{D} C N N$ rather than $2 \mathrm{D} C \mathrm{CNN}$ is used for processing sequence data in local block. (ii) A novel deep learning model, CRRNN for sequence to sequence learning is proposed; The model parameters are evaluated and 1D convolutional filter with one kernel is used for dimensionality reduction.

\section{Materials \\ Datasets}

A hybrid training set and five independent test datasets were used in this study. The training data is named TR12148 which consists of 12,148 polypeptide chains from the integration of the existing benchmark datasets TR5534 and TR6614. TR5534 was prepared by [17] that contains 5534 proteins. This benchmark dataset has been used to train the deep learning models including SCGSN [17], DCRNN [21], and conditioned CNN [22]. In fact, TR5534 was derived from the 6128 proteins of the CB513 dataset after sequence identity reduction. Dataset TR6614 contains 6614 non-homologous sequences produced using the PISCES Cull PDB server [27]. Protein sequences in TR6614 have a similarity less than $25 \%$, a resolution better than $3.0 \AA$ and an $\mathrm{R}$ factor of 1.0. The redundancy with test datasets was removed using cd-hit [28]. A detailed sequences list of TR6614 is given in Additional file 1 in supplemental information. We randomly selected 248 proteins as a validation dataset (VR248) and 240 proteins as test dataset (TS240) from TR12148, respectively, and used the remaining 11,700 proteins for training. The 3D structure files were downloaded from the RCSB Protein Data Bank (PDB).

Four public test datasets (named CB513, CASP10, CASP11, and CASP12) were used to evaluate the Q8 
and Q3 performance of our proposed model. CB513 is from [17]. CASP10, CASP11, and CASP12 are from the "Protein Structure Prediction Center". CASP10 contains 123 domain sequences extracted from 103 chains; CASP11 contains 105 domain sequences extracted from 85 chains; and CASP12 contains 40 chains. The total residues of the sequences from CASP10, CASP11, CASP12 and CB513 are 22041, 20498, 10526 and 87041 respectively. More details of the Q8 secondary structures in these datasets are listed in Table 1.

TR12148 is a dataset merging TR5534 and TR6614, and it contains 2,976,315 residues. The sequence lengths of the proteins in TR6614 range from 60 to 700 and the length range of the proteins in TR5534 is from 50 to 700 . Sequence lengths of the proteins in the test datasets are capped at 700 as well. If the length of a sequence from the test datasets is longer than 700 , the sequence is splitted into two sequences. The 700-residue length cut-off was chosen to provide a good balance between efficiency and coverage, given that the majority of the protein chains are shorter than 700 residues.

\section{Input features}

Four types of features, including a position-specific scoring matrix (PSSM), protein coding features, conservation scores, and physical properties, are used to characterize each residue in a protein sequence. To generate a PSSM, we ran PSI-Blast [29] to search the NCBI non-redundant database through three iterations with $E$-value $=0.001$. The physical property features [30] have been previously used for protein structure and property prediction $[19,31]$. These physical properties are: steric parameters (graph-shape index), polarizability, normalized van der Waals volume, hydrophobicity, isoelectric point, helix probability, and sheet probability. These specific values were downloaded from Meiler's study [30]. To ensure the network gradients decrease smoothly, these above 27 features were normalized by logistic function.
The 1-dimensional conservation score was computed by the method [32](1),

$$
R=\log 20+\sum_{i=1}^{20} Q_{i} \log Q_{i}
$$

Residue conversion was conducted according to amino acid frequency distribution in the corresponding column of a multiple-sequence alignment of homologous proteins. The score information in the PSSM was calculated from this probability. Residue score in the $i$-th column was calculated as follows [33]:

$$
S_{i}=\left[\ln \left(Q_{i} / P_{i}\right)\right] / \lambda_{u} .
$$

where $Q_{i}$ is a predicted probability that a properly aligned homologous protein has amino acid $i$ in that column, $P_{i}$ is the background probability [29], and $\lambda_{u}=0.3176 . Q_{i}$ is defined as $Q_{i}=\exp \left(S_{i} * \lambda_{u}\right) * P_{i}$.

The commonly used protein coding is an orthogonal coding. As Zhou's [17] scheme, the 22-dimensional coding vector is a sparse one-hot vector, only one of 22 elements is none-zero and a zero vector is no use for gradient optimization. Like description by [21], we adopted an embedding operation from natural-language processing to transform sparse sequence features into a denser representation. This embedding operation was implemented as a feed-forward neural network layer with an embedding matrix mapping a sparse vector into a denser 22-dimensional vector.

In our scheme, one residue is represented by 50 -dimensional features (20-dimensional PSSM, 7-dimensional physical properties, 1-dimensional conservation score and 22-dimensional protein coding information). The secondary structure labels are generated by DSSP [2]. Similar to Zhou's method [17], proteins shorter than 700 AA were padded with all-zero features and the corresponding outputs are labeled with "NoSeq". The advantage of padding these proteins is to enable the training of the model on GPU in batches.

\begin{tabular}{|c|c|c|c|c|c|c|c|c|c|c|c|c|c|}
\hline \multirow[t]{2}{*}{ Label } & \multirow[t]{2}{*}{ Types } & \multicolumn{2}{|l|}{ TR6614 } & \multicolumn{2}{|l|}{ TR5534 } & \multicolumn{2}{|l|}{ CB513 } & \multicolumn{2}{|l|}{ CASP10 } & \multicolumn{2}{|c|}{ CASP11 } & \multirow{2}{*}{$\begin{array}{l}\text { CASP12 } \\
\text { Count }\end{array}$} & \multirow[b]{2}{*}{$\%$} \\
\hline & & Count & $\%$ & Count & $\%$ & Count & $\%$ & Count & $\%$ & Count & $\%$ & & \\
\hline $\mathrm{H}$ & $\alpha$-helix & 517653 & 0.352 & 405560 & 0.345 & 26143 & 0.309 & 6544 & 0.297 & 6330 & 0.309 & 3550 & 0.337 \\
\hline B & $\beta$-bridge & 15321 & 0.010 & 12096 & 0.010 & 1180 & 0.014 & 227 & 0.010 & 221 & 0.011 & 113 & 0.011 \\
\hline E & $\beta$-strand & 321156 & 0.218 & 255887 & 0.218 & 17994 & 0.212 & 5225 & 0.237 & 5089 & 0.248 & 2223 & 0.211 \\
\hline G & 310 helix & 55994 & 0.038 & 46019 & 0.039 & 3132 & 0.037 & 797 & 0.036 & 716 & 0.035 & 320 & 0.030 \\
\hline I & $\pi$-helix & 281 & 0 & 209 & 0 & 30 & 0 & 5 & 0 & 0 & 0 & 0 & 0 \\
\hline $\mathrm{T}$ & Turn & 160753 & 0.109 & 132980 & 0.113 & 10008 & 0.118 & 2811 & 0.128 & 2299 & 0.112 & 1164 & 0.111 \\
\hline S & Bend & 118800 & 0.081 & 97298 & 0.083 & 8310 & 0.098 & 1780 & 0.081 & 1751 & 0.085 & 955 & 0.091 \\
\hline L & Coil & 282584 & 0.192 & 225493 & 0.192 & 17904 & 0.211 & 4652 & 0.211 & 4092 & 0.200 & 2201 & 0.209 \\
\hline All & & 1472542 & & 1175542 & & 84701 & & 22041 & & 20498 & & 10526 & \\
\hline
\end{tabular}

Table 1 Training and test data used in our work 


\section{Methods}

As illustrated in Fig. 1, our CRRNN model consists of four parts: a local block, three stacked bidirectional gated recurrent unit (BGRU, or BGRU block) layers, two residual connections, and two fully-connected layers. The local block capture local sequence features and feeds them to the first BGRU layer, and the residual network transfers data to the subsequent BGRU layers. In the BGRU block, two types of input data are concatenated and fed to the next BGRU layer. At the end of the fully connected layer, the softmax activation outputs the predicted results in either the 8- or 3-state category.

\section{Local block}

Extracting information from protein sequences by convolutional neural network has fast progressed [17, 20-22]. The application of the convolution operator is dependent upon input dimensionality [34]. Two-dimensional kernels are often used in a $2 \mathrm{D}$ spatial convolutional operator, whereas a $1 \mathrm{D}$ convolutional network is usually used for processing sequences. In the $1 \mathrm{D}$ domain, a kernel can be viewed as a filter capable of removing outliers to filter data or act as a feature detector. Here, we used a 1D CNN to model the local dependencies of adjacent amino acids. Given the sequence data

$$
X=\left(x_{1}, x_{2}, x_{3} \ldots x_{t-1}, x_{t}, x_{t+1} \ldots x_{n}\right),
$$

where $x_{i}=\left(x_{i 1}, x_{i 2}, \ldots x_{i j}, \ldots x_{i m}\right)$ is a feature vector of the $i$ th residue. Residue $x_{i}$ is context-dependent and strongly reliant on forward and backward information; however, the value space of feature $x_{i j}$ might differ from $x_{i k}$. Overall, residue orientation is convoluted by the $1 \mathrm{D} C \mathrm{NN}$ :

$$
h_{i}=f\left(W * x_{i: i+k-1}+b\right)
$$

where “" denotes the convolutional operation, and $k$ represents the kernel size. Considering that the minimum length of the second structure, the kernel sizes of CNN in local block are set to three and five. One-hundred filters were used separately, and a rectified linear unit function activates the network output. To capture more structure information, the original input data is concatenated with the convolutional network output. Compared with the kernel size of 7,11 [21] and $9 \times 24$ [22], our network parameters were smaller and they could effectively capture the local information.

\section{BGRU and BGRU block}

Protein structures are affected largely by long-range interactions between residues. Recurrent neural network (RNN) can model large-distance dependencies between amino acids. At a given time $T=t$, the recurrent neural network can remember information from past input, $x_{1}, x_{2}, x_{3} \ldots x_{t-1}$, and current input $x_{t}$. However, the output, $y_{t}$, might depend upon the contextual protein sequence. The BRNN [35] combines a RNN that moves forward through time beginning from the start of the sequence along with another RNN that moves backward through time beginning from the end of the sequence. In the BRNN, increased input over time is represented by $\vec{f}\left(x_{1}, x_{2}, x_{3}, \ldots, x_{t-1}\right)$, and the decreased input over time is represented by $\overleftarrow{f}\left(x_{t+1}, \ldots, x_{n}\right)$. Compared to RNN, the BRNN is more suitable for context-related applications, and its performance is better than unidirectional RNN.

The depth of a RNN makes the network difficult to train because of an exploding or vanishing gradient [36]. Long short-term memory (LSTM) [37], which consists of a variety of gate structures (forgotten gate, input gate, output gate and memory cell) can overcome with the vanishing

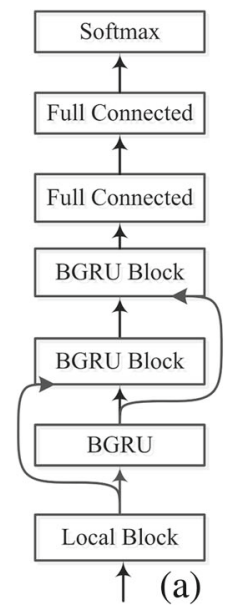

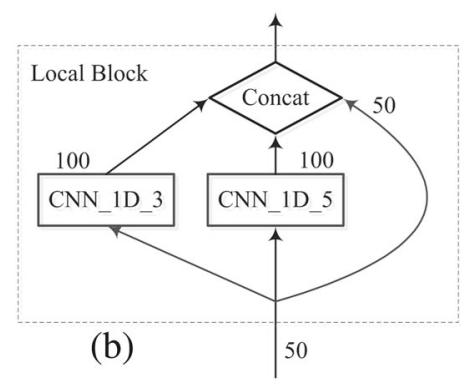

(b)

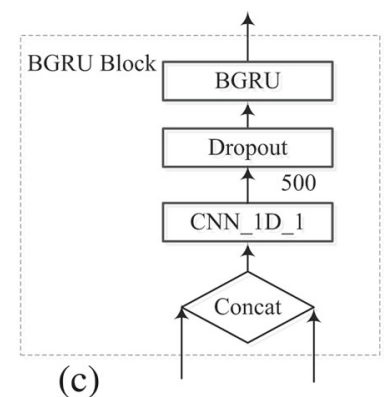

(c)

Fig. 1 a CRRNN overall architecture. b A local block comprising of two 1D convolutional networks with 100 kernels, and the concatenation (Concat) of their outputs with the original input data. c the BGRU block. The concatenation of input from the previous layer and before the previous layer is fed to the 1D convolutional filter. After reducing the dimensionality, the 500-dimensional data is transferred to the next BGRU layer 
gradient problem. Compared with a LSTM, gate recurrent units (GRU) achieved comparable performance, and required fewer parameters [36]. The details of GRU is described by the following formula (5):

$$
\begin{aligned}
& r_{t}=\sigma\left(W_{x r} x_{t}+W_{h r} h_{t-1}+b_{r}\right) \\
& z_{t}=\sigma\left(W_{x z} x_{t}+W_{h z} h_{t-1}+b_{z}\right) \\
& \tilde{h_{t}}=\tanh \left(W_{x h} x_{t}+W_{h h}\left(r_{t} \bigodot h_{t-1}\right)+b_{h}\right) \\
& h_{t}=z_{t} \odot h_{t-1}+\left(1-z_{t}\right) \odot \tilde{h}_{t}
\end{aligned}
$$

where $\sigma$ is the sigmoid function, $\odot$ represents an element-wise multiplier. $r_{t}, z_{t}, \tilde{h_{t}}$ and $h_{t}$ are the reset gate, update gate, internal memory cell activation vectors and output, respectively. We construct three BGRU layers in the CRRNN model. When the forward-computed result $F_{t}$ is merged with the backward result, $B_{t}$, merging computation in the first GRU layer is concatenated, and the others are summed, as formula (6):

$$
\begin{aligned}
& O_{t}^{1}=\operatorname{Concat}\left(F_{t}, B_{t}\right) \\
& O_{t}^{2,3}=F_{t}+B_{t} \\
& \text { s.t. } F_{t}=\left(\overleftrightarrow{h_{1}}, \vec{h}_{2}, \ldots, \overrightarrow{h_{t}}\right), \\
& \quad B_{t}=\left(\overleftarrow{h_{t}}, \overleftarrow{h}_{t+1}, \ldots, \overleftarrow{h_{n}}\right)
\end{aligned}
$$

In first BRNN layer, 250 units were used in the unidirectional RNN, and the dimensionality of the output was 500. In the 2nd and 3rd layer, 500 units were used in the unidirectional RNN. Based on the improved performance of the CNN model [25] using additive identity shortcuts between the outputs of the lower layers and the inputs to higher layers, which improved information flow throughout the network, Fig. 1c shows how we introduce this process into recurrent neural network. $h_{t}^{l}$ is the previous layer output and $h_{t}^{l-1}$ is the previous layer input. $I_{t}$, the concatenation of them will be fed to current hidden layer,

$$
\begin{aligned}
I_{t} & =\text { Concat }\left(h_{t}^{l}, h_{t}^{l-1}\right) \\
I_{t}^{\prime} & =f\left(W * I_{t}\right)
\end{aligned}
$$

To avoid the explosion caused by feature concatenation of the input from the previous layer, the BGRU block used the 1D CNN with one kernel to control the high dimensionality. Concatenating operation is not as same as the summing operation used in residual network, for it can reserve more information.

\section{Implementation details}

In our experiments, an Adam optimizing function was used for training the entire network of the default setting parameters. The default learning rate was initially set at 0.0004 with a decreasing step 0.0001 , whereas the validation accuracy did not increase after more than 10 epochs. The learning-rate threshold was set to 0.0001 . A cross-entropy loss function was used to train the model. Weight constraint of dropout $(\mathrm{p}=0.5)$ used to avoid overfitting were applied to the output filters before advancing to the next BGRU layer. The algorithm was enforced to complete when validation accuracy stopped increasing. When the model had iterated about 130 epochs, it converged and predictive performance stabilized. Our model was implemented in Keras, which is a publicly available deep-learning software. Weights in the CRRNN were initialized using default values, and the entire network was trained on a single NVIDIA GeForce GTX 1080 Ti GPU with $12 \mathrm{~GB}$ memory.

\section{Results and discussion}

\section{Performance for Q8 and Q3 prediction}

Our model, which was trained individually ten times using the TR12148 dataset, achieved a $73.3 \pm 0.4 \%$ accuracy on the TS240 test set. As an individual model, we performed validation on the CB513 benchmark and achieved a $71.4 \pm 0.2 \%$ accuracy, competitively matching that of the state-of-the-art method using the NCCNN ensemble model [22] and 1.1\% higher than the NCCNN single model. The single model of NCCNN was iterated at least 1000 epochs while our model converged after only 130 epochs. We also compared our model with other representative methods, such as MUFOLD-SS [23], DCRNN [21], DeepCNF [20], and GSN [17], and BLSTM [18].

Except that MUFOLD-SS are trained using 9000 proteins, most of them are trained on TR5534. We did re-implement Conditioned CNN and DCRNN and used TR12148 as the training data. As some errors were occurred in the re-implemented 2D CNN, we replaced 2D CNN with 1D CNN. The performance by the reimplemented DCRNN exceeded the original results. The performance by the re-implemented NCCNN is weaker than the original results. Details of precision and recall are shown in Tables 2 and 3. The overall performance is shown in Table 4. DCRNN2 was re-implemented by us and trained on TR12148.

For all of these methods, their prediction accuracies on the CASP10 dataset are higher than on the other datasets,

Table 2 Q8 predictive precision of individual secondary structures from CB513

\begin{tabular}{lllllll}
\hline Q8 Label & CRRNN & NCCNN & MUFOLD-SS & DCRNN2 & DCRNN & DeepCNF \\
\hline H & $\mathbf{0 . 8 6}$ & 0.841 & 0.855 & 0.863 & 0.832 & 0.849 \\
B & 0.466 & $\mathbf{0 . 6 7 6}$ & 0.571 & 0.571 & 0.554 & 0.433 \\
E & $\mathbf{0 . 7 9 7}$ & 0.767 & 0.764 & 0.768 & 0.753 & 0.748 \\
G & 0.466 & $\mathbf{0 . 4 8 7}$ & 0.413 & 0.419 & 0.429 & 0.49 \\
I & 0 & 0 & 0 & 0 & 0 & 0 \\
T & 0.556 & $\mathbf{0 . 5 7 7}$ & 0.572 & 0.562 & 0.559 & 0.53 \\
S & 0.494 & $\mathbf{0 . 5 4 8}$ & 0.522 & 0.509 & 0.518 & 0.487 \\
L & $\mathbf{0 . 6 0 3}$ & 0.565 & 0.586 & 0.571 & 0.573 & 0.571 \\
\hline
\end{tabular}

${ }^{a}$ Data is generated by our experiment

Boldface numbers indicate best performance 
Table 3 Recall of individual secondary structures is compared on CB513 for Q8 prediction

\begin{tabular}{|c|c|c|c|c|c|c|}
\hline Q8 Label & CRRNN & NCCNN & MUFOLD-SS & DCRNN2a & DCRNN & DeepCNF \\
\hline $\mathrm{H}$ & 0.926 & 0.932 & 0.920 & 0.920 & 0.933 & 0.904 \\
\hline B & 0.081 & 0.041 & 0.071 & 0.003 & 0.026 & 0.026 \\
\hline E & 0.831 & 0.821 & 0.815 & 0.841 & 0.828 & 0.833 \\
\hline G & 0.371 & 0.285 & 0.364 & 0.359 & 0.252 & 0.26 \\
\hline I & 0 & 0 & 0 & 0 & 0 & 0 \\
\hline T & 0.555 & 0.524 & 0.549 & 0.539 & 0.522 & 0.528 \\
\hline S & 0.332 & 0.24 & 0.290 & 0.258 & 0.249 & 0.255 \\
\hline L & 0.658 & 0.69 & 0.662 & 0.658 & 0.652 & 0.657 \\
\hline
\end{tabular}

${ }^{a}$ Data is generated by our experiment

Boldface numbers indicate best performance

and the accuracies on the CASP12 dataset are lower. One reason is that the profiles of CASP10 is extracted from the NCBI NR database which represent the sequences more precisely. CASP12 contains more hard cases and the PSSM profiles are not as good as those in CASP10 or CB513.

Tables 2 and 3 show the model performance on individual secondary structures. F1-score, which corresponds to the harmonic means of precision and recall, is also compared in Table 5. Macro_F1 [38] represents the unweighted mean of all the categories, whereas micro_F1 represents the averages of global total true positives; therefore, this indicator has the same value as the accuracy.

$$
\begin{aligned}
& F 1=\frac{2 *(\text { precision } * \text { recall })}{(\text { precision }+ \text { recall })} \\
& \text { macro_ } F_{1}=\frac{1}{n} \sum_{i=1}^{n} F_{1 i}
\end{aligned}
$$

Table 4 A comparison of the Q8 accuracy(\%) on CB513, CASP10, CASP11 and CASP12 between CRRNN and other state-of-the-art

\begin{tabular}{|c|c|c|c|c|}
\hline method & CB513 & CASP10 & CASP11 & CASP12 \\
\hline GSN & 66.4 & - & - & - \\
\hline BLSTM & 67.4 & - & - & - \\
\hline DeepCNF & 68.3 & 71.8 & $71.7^{b}$ & $0.694^{b}$ \\
\hline DCRNN & 69.7 & - & - & - \\
\hline DCRNN2a & 70.4 & 73.9 & 71.2 & 68.8 \\
\hline NCCNN & 70.3 & - & - & - \\
\hline NCCNN ${ }^{a}$ & 71.4 & - & - & - \\
\hline MUFOLD-SS ${ }^{b}$ & 70.5 & 74.2 & 71.6 & 69.5 \\
\hline CRRNN & $71.4 \pm 0.2$ & $73.8 \pm 0.5$ & $71.6 \pm 0.7$ & $68.7 \pm 0.8$ \\
\hline eCRRNN ${ }^{a}$ & 74 & 76.3 & 73.9 & 70.7 \\
\hline
\end{tabular}
methods

aindicates ensemble model

${ }^{b}$ Data is generated by our experiment

Boldface numbers indicate best performance
The F1 score related to individual secondary structure for our model exceeded those by the other methods, indicating that our model exhibited better predictive ability. The macro_F1 score of our model was also better than those by the other methods.

To validate the generalization capability of our model, independent test datasets CASP10, CASP11, and CASP12 were used. The performance results are reported in Table 4. The performance for CASP10, CASP11, and CASP12 by NCCNN were not supplied.

By the same way as [20], we mapped 8-state labels to 3-state labels: $\mathrm{H}$ (8-state) was mapped to $\mathrm{H}$ (3-state), $\mathrm{E}$ (8-state) was mapped to $\mathrm{E}$ (3-state) and others (8-state) were mapped to $\mathrm{C}(3$-state). Q3 predictive performance was compared with those by DCRNN and DeepCNF on Table 6. The Q3 accuracy on the CB513 dataset was $85.3 \pm 0.4 \%$, which was $1.5 \%$ higher than the state-of-theart methods [21]. The predictive accuracy of our model on CASP10, CASP11 and CASP12 were $86.1 \pm 0.6 \%$, $84.2 \pm 0.5 \%$ and $82.6 \pm 1.2 \%$ respectively, and most of these were higher than the compared methods.

Another newest Q3 prediction tool SPIDER3 [19] using a two-layered BLSTM was proposed, wherein $\mathrm{H}, \mathrm{G}$, and I (8-state) are mapped to $\mathrm{H}$ (3-state), $\mathrm{E}$ and $\mathrm{B}$ (8-state) are mapped to $E$, and others (8-state) are mapped to C. Similarly, we trained our model and tested it on the TS1199 dataset [19], achieving $85.5 \%$ accuracy, which was higher than SPIDER3 (84.5\%) and SPIDER2 (81.8\%). Figure 2 compares the accuracy of secondary structure prediction at individual amino acid levels with SPIDER3 and SPIDER2, indicating higher accuracies than both at $82 \%$.

\section{Ensemble learning and case study}

In order to further evaluate the model generalization capability, an ensemble of ten independently trained models (named eCRRNN) is constructed. The outputs of the ensemble model are derived by averaging the individual predicted probabilities over the secondary structure labels (Eq. 9).

$$
y=\operatorname{argmax}\left(\frac{1}{N} \sum_{i=1}^{N} p_{i}\right)
$$

$p_{i}$ is the output probability of constituent model and the model has been trained independently. Ensemble methods can obtain better predictive performance that could be obtained from any of the constituent predictor independently [39]. Prediction of eCRRNN achieved 74\%, $76.3 \%, 73.9 \%$, and $70.7 \%$ Q8 accuracy on the CB513, CASP10, CASP11, and CASP12 datasets, respectively. The Q8 prediction performance is improved by $2.6 \%, 2.5 \%$ $2.3 \%$ and $2 \%$ on CB513, CASP10, CASP11 and CASP12 
Table 5 F1 score of individual secondary structure labels using CB513

\begin{tabular}{|c|c|c|c|c|c|c|c|c|}
\hline Q8 Label & CRRNN $^{a}$ & CRRNN & $\mathrm{NCCNN}^{\mathrm{a}}$ & NCCNN & MUFOLD-SS & DCRNN2 & DCRNN $^{a}$ & DeepCNF \\
\hline $\mathrm{H}$ & 0.903 & 0.892 & 0.889 & 0.884 & 0.886 & 0.891 & 0.880 & 0.876 \\
\hline B & 0.138 & 0.139 & 0.089 & 0.077 & 0.000 & 0.006 & 0.050 & 0.049 \\
\hline E & 0.834 & 0.814 & 0.805 & 0.793 & 0.789 & 0.803 & 0.789 & 0.788 \\
\hline G & 0.463 & 0.413 & 0.374 & 0.360 & 0.387 & 0.387 & 0.317 & 0.340 \\
\hline 1 & 0 & 0 & 0 & 0 & 0 & 0 & 0 & 0 \\
\hline $\mathrm{T}$ & 0.594 & 0.555 & 0.565 & 0.549 & 0.561 & 0.550 & 0.540 & 0.529 \\
\hline S & 0.433 & 0.397 & 0.343 & 0.334 & 0.373 & 0.342 & 0.336 & 0.335 \\
\hline L & 0.660 & 0.629 & 0.631 & 0.621 & 0.622 & 0.611 & 0.610 & 0.611 \\
\hline macro-F1 & 0.503 & 0.480 & 0.462 & 0.452 & 0.452 & 0.449 & 0.440 & 0.441 \\
\hline micro_F & 0.74 & 0.714 & 0.714 & 0.704 & 0.705 & 0.704 & 0.697 & 0.683 \\
\hline
\end{tabular}

${ }^{a}$ indicates ensemble model

Boldface numbers indicate best performance

respectively. We conducted analysis on the performance for the individual labels in CB513. Predictive accuracies of $\mathrm{H}$ type, $\mathrm{E}$ type and $\mathrm{L}$ type have been improved by $0.9 \%, 3.6 \%$ and $3.9 \%$ respectively. The secondary structures are imbalanced data and the majority labels are $\mathrm{H}, \mathrm{E}$ and $\mathrm{L}$. The ensemble model has effectively improved the classification accuracy for the major categories.

The precision and recall performance on the CB513 dataset are list in Table 7, and the F1 score, macro_F1, and micro_F1 are compared in Table 5. The F1 score for individual secondary structure prediction using our ensemble model was better than that of a NCCNN ensemble model. The predictive details on the CASP10, CASP11, and CASP12 datasets are listed in Table 8. We also validated its generalization on Q3 prediction and achieved 87.3\%, $87.8 \%, 85.9 \%$ and $83.7 \%$ on CB513, CASP 10 , CASP 11 , and CASP12. Both of the Q8 and Q3 prediction results are better than the state-of-the-art.

The $P$-value of significance test between CRRNN and MUFOLD-SS is 5.31E-7 $(<0.005)$; The $P$-value of difference between eCRRNN and MUFOLD-SS is 6.93E-15; and

Table 6 Q3 accuracy(\%) comparison on CB513 and CASP datasets

\begin{tabular}{lllll}
\hline Method & CASP10 & CASP11 & CASP12 & CB513 \\
\hline PSIPRED & 81.2 & 80.7 & $80.5^{\mathrm{a}}$ & 79.2 \\
JPRED & 81.6 & 80.4 & $78.8^{\mathrm{a}}$ & 81.7 \\
DeepCNF & 84.4 & 84.7 & $83.2^{\mathrm{a}}$ & 82.3 \\
DCRNN & - & - & - & 84 \\
NCCNN & - & - & - & - \\
MUFOLD-SS & 84.3 & 82.3 & 81.1 & 82.7 \\
CRRNN & $86.1 \pm 0.6$ & $84.2 \pm 0.5$ & $82.6 \pm 1.2$ & $85.3 \pm 0.4$ \\
eCRRNN & $\mathbf{8 7 . 8}$ & $\mathbf{8 5 . 9}$ & $\mathbf{8 3 . 7}$ & $\mathbf{8 7 . 3}$ \\
\hline
\end{tabular}

aData is generated by our experiment

Boldface numbers indicate best performance the significance test between CRRNN and eCRRNN is at the 0.0047 level.

Segment of OVerlap(SOV) score has been used to evaluate the predicted protein secondary structures comparing with the native secondary structures. If the predictive structure segments match more native structures, SOV score will more higher. We calculate the SOV'99 score [40] using the SOV_refine [41] tool which measures how well the native and the predicted structure segments match. As shown in Table 9, in terms of SOV score on CB513, CASP10, CASP11 and CASP12, eCRRNN obtained 72.5\%, $74.7 \%, 72.2 \%$ and $68.4 \%$ respectively. SOV scores on constituent secondary structure are also listed in Table 9. The comparison of SOV scores on CASP12 using eCRRNN, DeepCNF and MFOLD-SS is shown in Fig. 3. On the

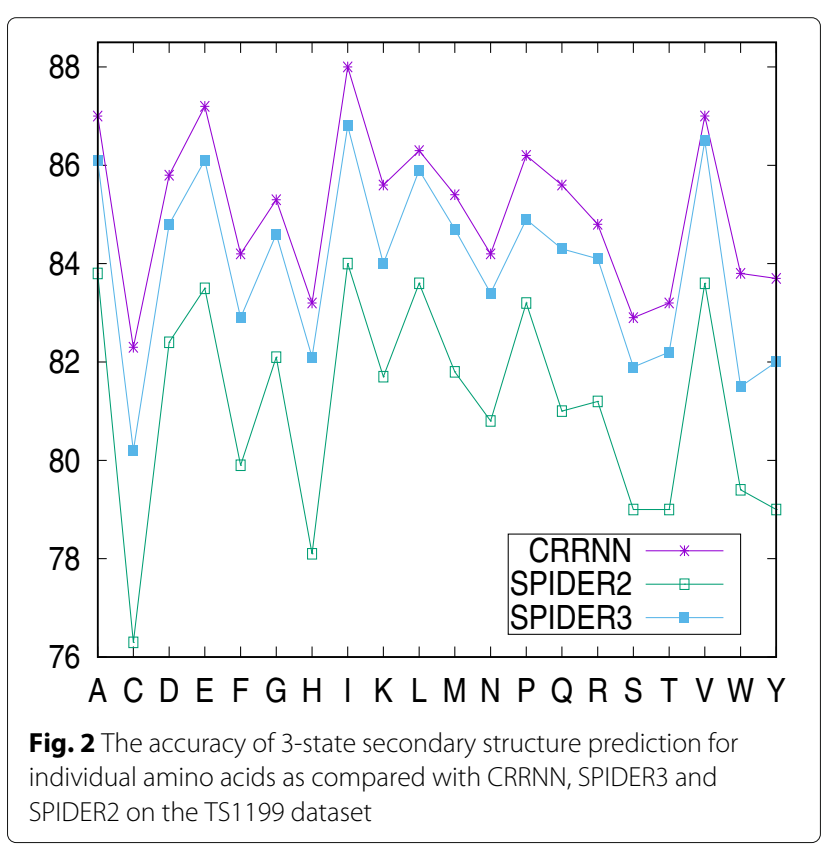


Table 7 Q8 prediction using the ensemble model on the CB513 dataset

\begin{tabular}{|c|c|c|c|c|c|c|c|c|}
\hline \multirow[t]{2}{*}{ Q8 Label } & \multicolumn{4}{|l|}{ precision } & \multicolumn{4}{|l|}{ recall } \\
\hline & eCRRNN* & CRRNN & $\mathrm{NCCNN}^{\mathrm{a}}$ & DCRNN $^{a}$ & eCRRNN ${ }^{a}$ & CRRNN & $\mathrm{NCCNN}^{\mathrm{a}}$ & $\mathrm{DCRNN}^{\mathrm{a}}$ \\
\hline $\mathrm{H}$ & 0.872 & 0.860 & 0.846 & 0.832 & 0.935 & 0.926 & 0.936 & 0.933 \\
\hline B & 0.582 & 0.466 & 0.786 & 0.554 & 0.078 & 0.081 & 0.047 & 0.026 \\
\hline E & 0.804 & 0.797 & 0.776 & 0.753 & 0.867 & 0.831 & 0.837 & 0.828 \\
\hline G & 0.554 & 0.466 & 0.528 & 0.429 & 0.398 & 0.371 & 0.29 & 0.252 \\
\hline । & 0 & 0 & 0 & 0 & 0 & 0 & 0 & 0 \\
\hline T & 0.603 & 0.556 & 0.591 & 0.559 & 0.586 & 0.555 & 0.542 & 0.522 \\
\hline S & 0.563 & 0.494 & 0.621 & 0.518 & 0.352 & 0.332 & 0.237 & 0.249 \\
\hline L & 0.626 & 0.603 & 0.570 & 0.573 & 0.697 & 0.658 & 0.707 & 0.652 \\
\hline
\end{tabular}

a indicates ensemble model

Boldface numbers indicate best performance

structure types B and G, the performance of eCRRNN is slightly weaker than that of MFOLD-SS. In a large number of continuous secondary structures, the performance of eCRRNN is better. Table 10 lists the detailed scores on Q3 prediction. We also compared predictive SOV score on CASP12 with JPRED, DeepCNF and MFOLD-SS, and the specific scores are listed in Table 11. Although the overall SOV score of our method is just $0.9 \%$ better than DeepCNF, the SOV score on structure $\mathrm{C}$ by our method is $74.1 \%, 8.3 \%$ better than DeepCNF. These SOV scores indicate that our method can match more continuous segments.

Port 5 [16] is the latest release of one of the best performing secondary structure predictor. The sequences of more than $40 \%$ of the similarity with Port 5 training dataset were removed, then the four public datasets are used as validating benchmark. The Q8 prediction accuracy using Port 5 is $74 \%, 76.3 \%, 74.2 \%$, and $70.9 \%$ respectively on CB513, CASP10, CASP11 and CASP12. The Q8 prediction accuracy using eCRRNN is $74.2 \%, 76.5 \%$, $73.8 \%$, and $70 \%$. The SOV score measured on Port 5 is $71.3 \%, 73.9 \%, 71.8 \%$ and $67.9 \%$. The SOV score measured

Table 8 Details of Q8 accuracy on the CASP10, CASP11, and CASP12 datasets predicted by an ensemble model of CRRNN

\begin{tabular}{|c|c|c|c|c|c|c|}
\hline \multirow[t]{2}{*}{ Q8 Label } & \multicolumn{2}{|l|}{ CASP10 } & \multicolumn{2}{|l|}{ CASP11 } & \multicolumn{2}{|l|}{ CASP12 } \\
\hline & precision & recall & precision & recall & precision & recall \\
\hline $\mathrm{H}$ & 0.894 & 0.925 & 0.867 & 0.931 & 0.853 & 0.926 \\
\hline B & 0.758 & 0.110 & 0.607 & 0.077 & 0.333 & 0.027 \\
\hline E & 0.829 & 0.868 & 0.796 & 0.864 & 0.746 & 0.837 \\
\hline G & 0.580 & 0.403 & 0.541 & 0.313 & 0.389 & 0.278 \\
\hline I & 0 & 0 & 0 & 0 & 0 & 0 \\
\hline $\mathrm{T}$ & 0.672 & 0.670 & 0.596 & 0.588 & 0.547 & 0.508 \\
\hline S & 0.561 & 0.366 & 0.523 & 0.327 & 0.490 & 0.263 \\
\hline L & 0.639 & 0.722 & 0.616 & 0.658 & 0.578 & 0.615 \\
\hline
\end{tabular}

on eCRRNN is $72.9 \%, 74.9 \%, 72.6 \%$ and $67.6 \%$. Although the prediction accuracy of Port 5 on casp 12 is higher than our method, it is almost the same with respect to the SOV score. The other SOV scores on our method are all better than those of Port 5 . These results show that eCRRNN could obtain more meaningful secondary structure predictions.

Specifically, proteins of length $\geq 400 \mathrm{AA}$ in the CB513 dataset were 20.The performance of MUFOLD-SS and DCRNN2 is $67.12 \%, 67.34 \%$. Our ensemble model achieved $72.49 \%$ accuracy on these proteins, which demonstrate the model effectiveness on capturing longrange information. The detailed performance is compared on Fig. 4.

Two examples are used to illustrate our model performance, with the predicted results from an ensemble CRRNN(eCRRNN) model, DCRNN2 and MUFOLDSS. A protein, T0786 (PDB-ID 4QVU), selected from

Table 9 SOV'99 scores of Q8 prediction using eCRRNN on 4 datasets: CB513, CASP10, CASP11 and CASP12

\begin{tabular}{|c|c|c|c|c|}
\hline Type & CB513 & CASP10 & CASP11 & CASP12 \\
\hline $\mathrm{SOV}_{L}$ & 0.611 & 0.629 & 0.595 & 0.538 \\
\hline $\mathrm{SOV}_{H}$ & 0.929 & 0.924 & 0.908 & 0.907 \\
\hline $\mathrm{SOV}_{T}$ & 0.599 & 0.67 & 0.605 & 0.531 \\
\hline $\mathrm{SOV}_{E}$ & 0.882 & 0.884 & 0.86 & 0.829 \\
\hline $\mathrm{SOV}_{\mathrm{S}}$ & 0.351 & 0.362 & 0.327 & 0.256 \\
\hline $\mathrm{SOV}_{B}$ & 0.078 & 0.11 & 0.077 & 0.027 \\
\hline $\mathrm{SOV}_{G}$ & 0.419 & 0.416 & 0.339 & 0.297 \\
\hline $\mathrm{SOV}_{1}$ & 0 & 0 & 0 & 0 \\
\hline SOV & 0.725 & 0.747 & 0.722 & 0.684 \\
\hline $\mathrm{SOV}_{\text {mean }}$ & 0.723 & 0.740 & 0.738 & 0.698 \\
\hline
\end{tabular}




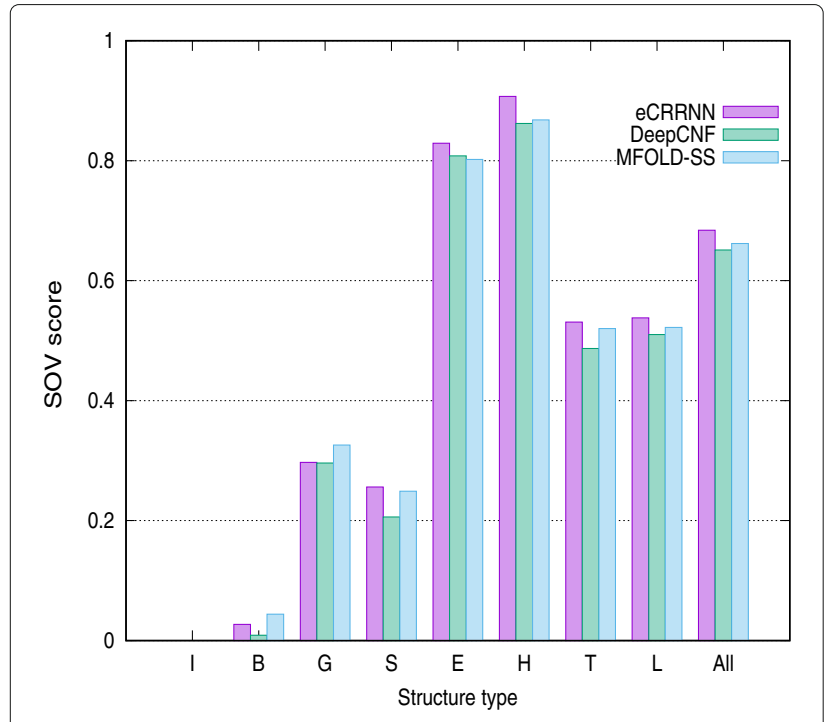

Fig. 3 The SOV score comparison of Q8 prediction on CASP12 dataset using eCRRNN, DeepCNF and MFOLD-SS. I, B, G, S, E, H, H, T, L represent the prediction SOV score on a individual secondary structure type respectively. "All" represents the SOV score on CASP12 dataset

the CASP11 dataset has 264 residues. The known secondary structure residues total only 217 AA (from residue 37 to 253). The native $3 \mathrm{D}$ structure is described in Fig. 5. The predictive accuracy according to DCRNN2, MUFOLD-SS and eCRRNN was $72.4 \%, 68.2 \%$, and $91.7 \%$. The comparison between native structure and predicted structure is described in Fig. 6. The results suggested that our model sufficiently captured continuous structure information.

The 3D structure of another protein (PDB: 6CPP) selected from the CB513 dataset is shown in Fig. 7 and represents an oxidoreductase of 414 residues (only 405 residues with known structures). Predictive accuracy by DCRNN2, MUFOLD-SS, and eCRRNN was $75.3 \%$, 76\%, and $88.4 \%$, respectively. Detailed prediction results are shown in Fig. 8. The accuracy of maximum continuous predicted structure from eCRRNN is 83AA. These results also indicate that our model was effective for long-chain

Table 10 SOV'99 scores of Q3 prediction using eCRRNN on 4 datasets: CB513, CASP10, CASP11 and CASP12

\begin{tabular}{lllll}
\hline Type & CB513 & CASP10 & CASP11 & CASP12 \\
\hline SOV $_{H}$ & 0.917 & 0.919 & 0.922 & 0.884 \\
SOV $_{E}$ & 0.859 & 0.868 & 0.835 & 0.798 \\
SOV $_{C}$ & 0.769 & 0.813 & 0.778 & 0.741 \\
SOV & 0.829 & 0.855 & 0.833 & 0.797 \\
SOV $_{\text {mean }}$ & 0.842 & 0.851 & 0.850 & 0.817 \\
\hline
\end{tabular}

$\mathrm{SOV}_{H}, \mathrm{SOV}_{E}$ and $\mathrm{SOV}_{C}$ represent the prediction SOV score on a constituent secondary structure type $\mathrm{H}, \mathrm{E}$ and $\mathrm{C}$ respectively. SOV represents the SOV score on different dataset and $\mathrm{SOV}_{\text {mean }}$ is mean value of the SOV score on sequence level
Table 11 SOV'99 scores of Q3 prediction on CASP12 using recently predicting methods are compared

\begin{tabular}{lllll}
\hline Method & SOV $_{H}$ & SOV $_{E}$ & SOV $_{C}$ & SOV \\
\hline JPRED & 0.827 & 0.747 & 0.676 & 0.737 \\
DeepCNF & 0.873 & 0.799 & 0.658 & 0.788 \\
MFOLD-SS & 0.879 & $\mathbf{0 . 8 1 4}$ & 0.594 & 0.715 \\
eCRRNN & $\mathbf{0 . 8 8 4}$ & 0.798 & $\mathbf{0 . 7 4 1}$ & $\mathbf{0 . 7 9 7}$ \\
\hline
\end{tabular}

$\mathrm{SOV}_{H}, \mathrm{SOV}_{E}$ and SOV $\mathrm{C}$ represent the prediction SOV score on a constituent secondary structure type $\mathrm{H}, \mathrm{E}$ and $\mathrm{C}$ respectively. SOV represents the SOV score on CASP12 dataset

Boldface numbers indicate best performance

protein structures. From the two cases, isolated residues which are not as same as previous and backward residue were not properly predicted, for the captured information is strongly depended on context residues.

\section{Ablation learning}

The total parameters of our model were about 7.74 million. The feature values provided by TR5534 with 50 dimensional features were 58.777 million and the ratio of training features to model parameters was 7.6:1. The ratio of features on TR12148 to the model parameters was about $16.4: 1$, which is bigger than the practical requirement (10:1).

We trained the model using the TR5534 dataset. After about 55 epochs, the predictive accuracy for CB513 dataset decreased and the loss became increasing. The model encountered overfitting problem as Fig. 9 illustrated. The model with two BGRU layers, which were capable of reducing about 1 million parameters, was also

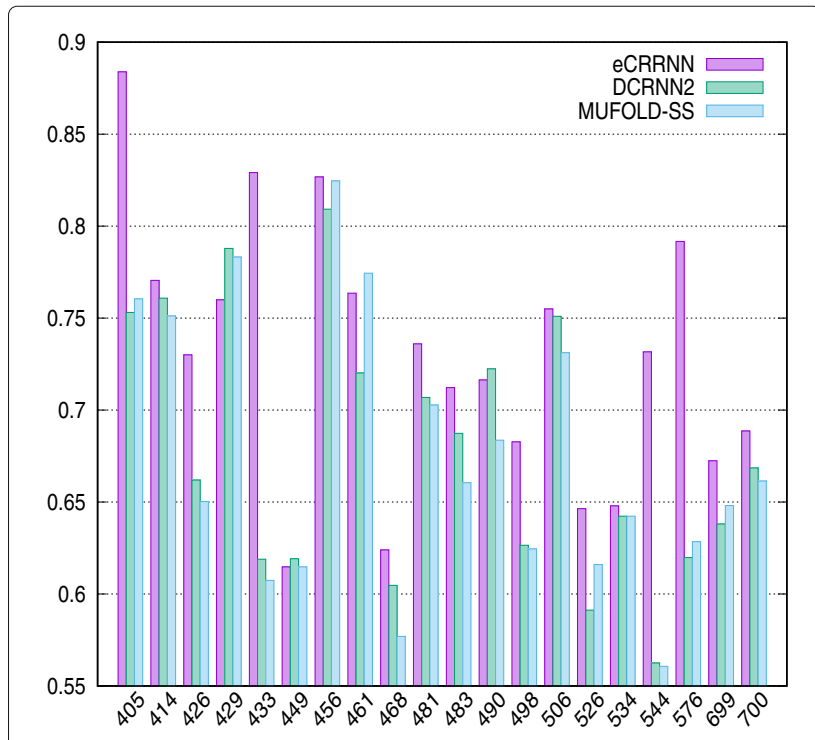

Fig. 4 The prediction comparison of 20 sequences from CB513 dataset which sequence length is more than 400AA 


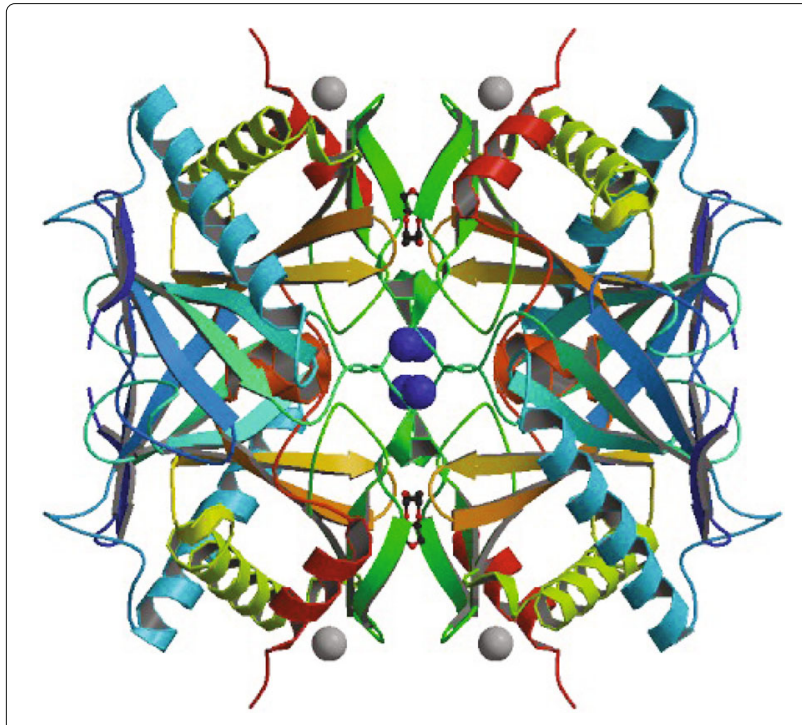

Fig. 5 Protein 4QVU native 3D structure

trained using TR5534. And the prediction for CB513 dataset shows that model's generalization was decreased. Table 12 lists the predictive performance on CB513 when model was trained using TR5534, TR6614 and TR12148. Figure 10 shows the model loss variation trained using TR12148. The training error increased along with increases in the size of the training set, because larger datasets are harder to fit. Meanwhile, the loss error of CB513 dataset was decreased, for fewer incorrect hypotheses were consistent with the training data.

To discover important factors related to the optimal utilization of our proposed model, we evaluated alternative

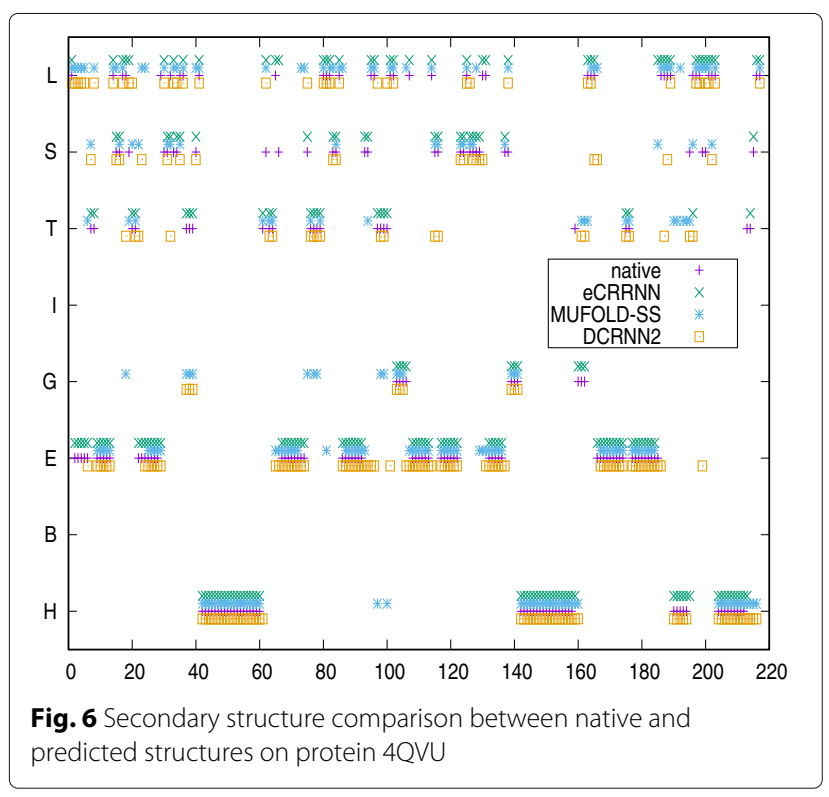

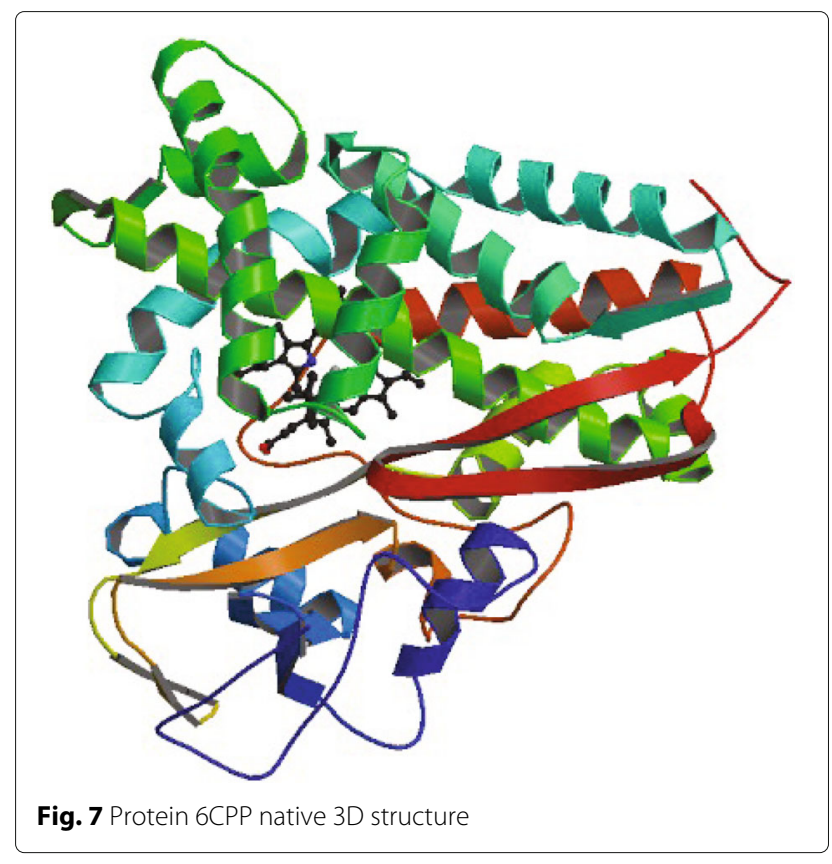

architectures by removing individual components. We specifically tested the performance of models without a local block or residual connections, as well as the models with 2-layer BGRUs where the input vectors were 42-dimensional features.

The test results on CB513 (Table 13) show that input features were slightly affected, and that the most important constituent was the BRNN. When input features comprised a 20-dimensional PSSM and 22-dimensional

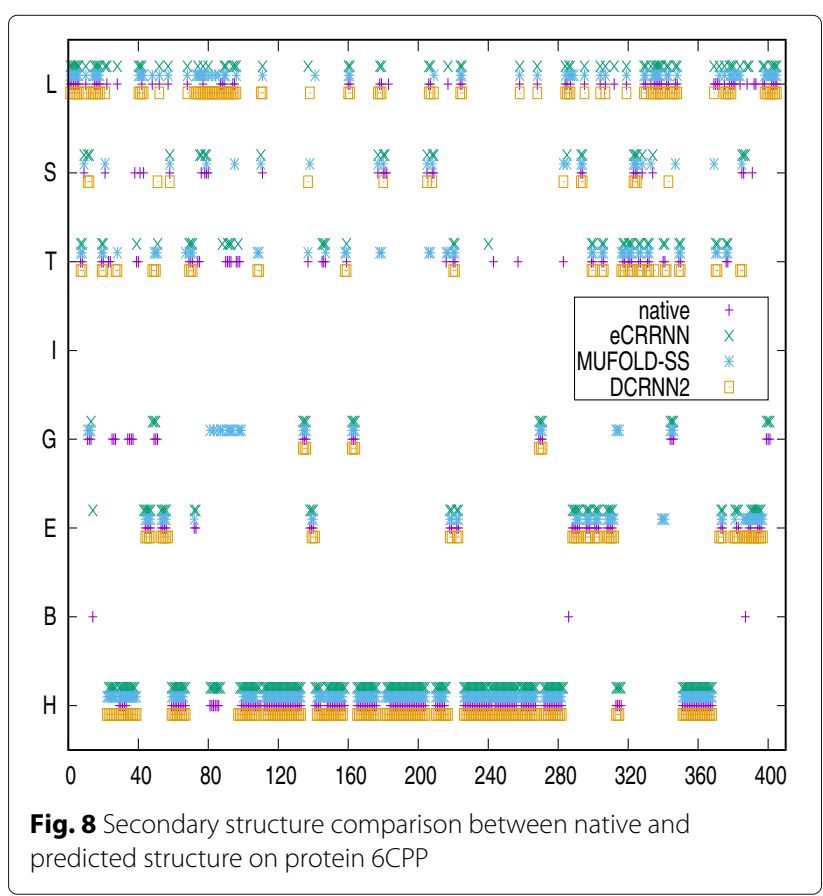




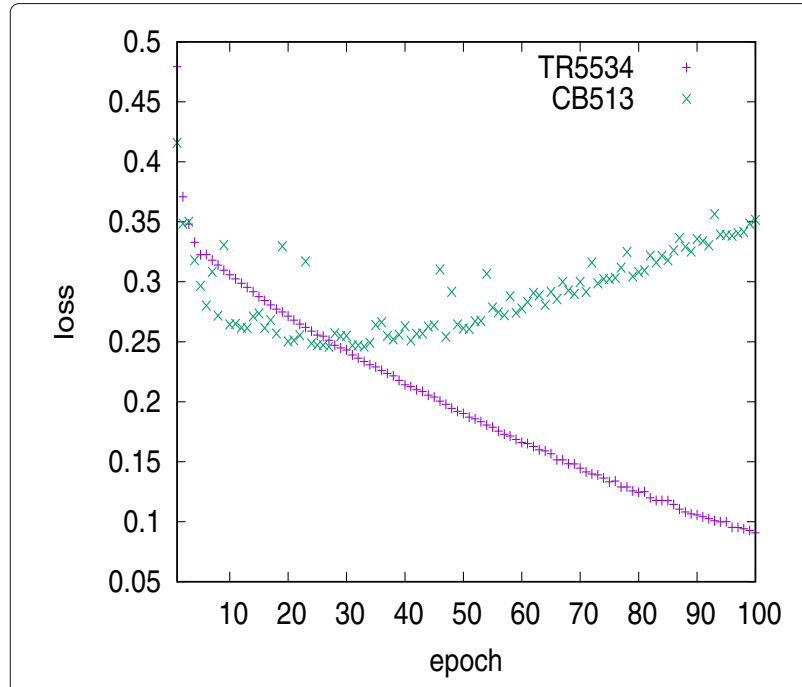

Fig. 9 Model loss variation trained using TR5534. About 55 epochs, loss on CB513 dataset stopped decreasing and the model became overfitting

protein coding, the performance just decreased by $0.1 \%$. When the recurrent neural network was constructed by unidirectional GRU, the performance dropped to $67.2 \%$. Protein structure is particularly depended upon context residues; therefore, the unidirectional GRU network was ineffective at capturing contextual dependencies. Regarding the number of stacked BGRU layers, the performance of the network architecture with 1-layer was poor. When the staked layers were increased to two layers, the performance increased to $70.5 \%$, and three-layer networks increased further to $71.4 \%$ accuracy. Increases in the stacked BRNN layers allowed the capture of more longrange information. Furthermore, the use of residual network indicated that shortcut connections between BRNN layers were essential for improving BRNN generalization. Without the residual network, accuracy dropped to $70.7 \%$. These results are not presented on a model scale. Upon replacement of the BRNN hidden node with a LSTM, the model parameters increased to 9.99 million while the accuracy dropped to $70.2 \%$, because the model had become overfitted and had not been adequately trained. When the 1D CNN filter with one kernel was removed, performance improved slightly improved, but 1.73 million

Table 12 Model-performance comparison using different training sets against CB513

\begin{tabular}{lll}
\hline Training set & Model & Accuracy(\%) \\
\hline TR5534 & CRRNN with 3-layer BGRU & 69.6 \\
TR5534 & CRRNN with 2-layer BGRU & 69.0 \\
TR6614 & CRRNN with 3-layer BGRU & 70.6 \\
TR12148 & CRRNN with 3-layer BGRU & 71.4 \\
\hline
\end{tabular}

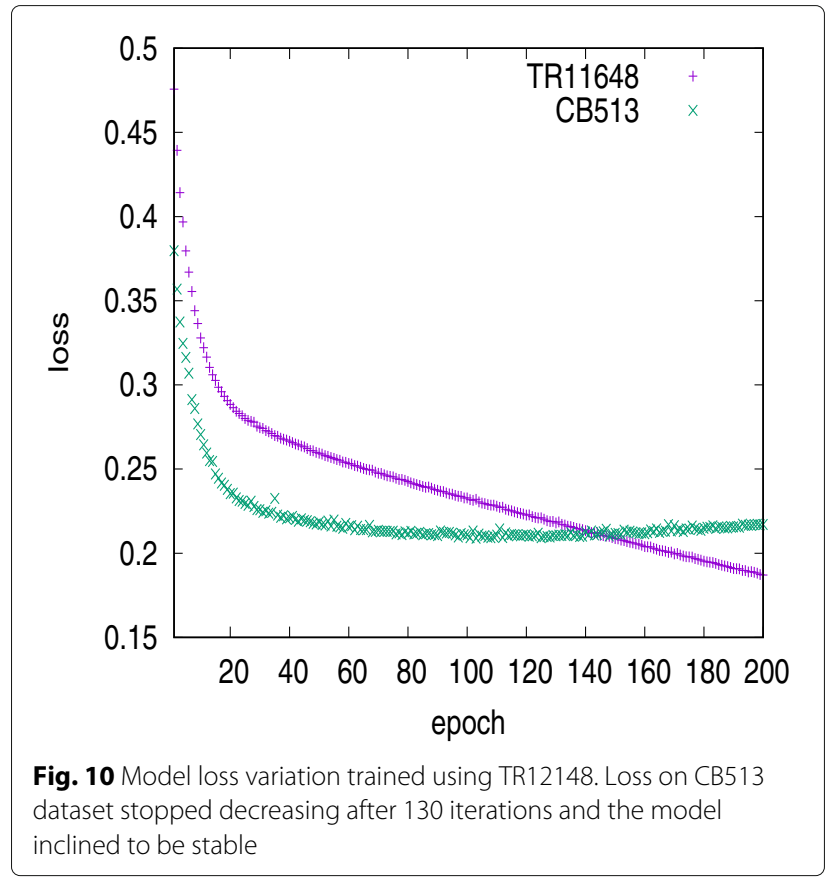

parameters increased. These results indicated that the $1 \mathrm{D}$ CNN with 1 kernel effectively controlled model dimensionality without reducing model generalization. And the local block improved also overall accuracy.

\section{Conclusion}

The CNN was successful at feature extraction, and the RNN was successful at sequence processing. Given that the residual network ImageNet [25] stacked 152 layers of convolutional neural network, we proposed a novel sequence-to-sequence deep learning model (CRRNN) for protein secondary structure prediction. Here, 1D $\mathrm{CNN}$ and original data were constructed into a local block to capture adjacent amino acid information. The residual network connected the interval BGRU network to improve modeling long-range dependencies. Our

Table 13 Comparison of different model's generalization performance

\begin{tabular}{ll}
\hline Model & Accuracy(\%) \\
\hline CRRNN & $71.4 \pm 0.2$ \\
Without ResNet & $70.7 \pm 0.2$ \\
3-layer with BLSTM & $70.2 \pm 0.2$ \\
Without local bolck & $71.1 \pm 0.3$ \\
Without 1D one kernel CNN filter & $71.5 \pm 0.2$ \\
With 2-layer BGRU & $70.5 \pm 0.1$ \\
Unidirectional GRU & 67.2 \\
With 1-layer BGRU & 69.5 \\
CRRNN with 42dim features input & $71.3 \pm 0.2$ \\
\hline
\end{tabular}


ensemble model was more generalizable, and the overall performance exceeded the performance by the state-ofthe-art methods for both 8- and 3-state prediction. The model can also be used to predict other sequence-labeling problems and is not limited to biological problems.

\section{Additional file}

Additional file 1: The file lists 6614 protein sequences PDB-ID which were used training in our work. (DOCX $38 \mathrm{~kb}$ )

\section{Abbreviations}

BGRU: Bidirectional gated recurrent unit; BLSTM: Bidirectional long short-term memory; BRNN: Bidirectional recurrent neural network; CNN: Convolutional neural network; CRRNN: Convolutional, residual, and recurrent neural network; eCRRNN: Ensemble of convolutional, residual, and recurrent neural network; GRU: Gated recurrent units; LSTM: Long short-term memory; NCCNN: Next-step conditioned convolutional neural network; PDB: Protein data bank; PSSM: Position-specific scoring matrix; RNN: Recurrent neural network

\section{Acknowledgements}

We wish to thank Zhen Li, Sheng Wang and Jian Zhou for discussion and consultation about datasets.

\section{Funding}

This work is supported in part by National Natural Science Foundation of China (No.61170125), the Special Program for Applied Research on Super Computation of the NSFC-Guangdong Joint Fund (the second phase) and Natural science research project of Anhui Provincial Department of Education (No.KJ2018A0383). The fundings have no role in the design of the study and collection, analysis, and interpretation of data and writing the manuscript.

\section{Availability of data and materials}

The secondary structure prediction tool of CRRNN, PDB-ID set of TR6614 and testing datasets can be download from http://web.suda.edu.cn/qiang/ecrrnn. html.

\section{Authors' contributions}

BZ and QL conceived the study; BZ performed the data collection, training, prediction and analysis; $\mathrm{L}$ and $\mathrm{QL}$ redesigned the experiment and data analysis; BZ, QL and JL wrote the paper; All authors contributed to the revised and approved the final manuscript.

\section{Ethics approval and consent to participate}

Not applicable.

\section{Competing interests}

The authors declare that they have no competing interests.

\section{Publisher's Note}

Springer Nature remains neutral with regard to jurisdictional claims in published maps and institutional affiliations.

\section{Author details \\ ${ }^{1}$ School of Computer Science and Technology, Soochow University, Suzhou, China. ${ }^{2}$ School of Computer and Information, and the University Key Laboratory of Intelligent Perception and Computing of Anhui Province, Anqing Normal University, 246011 Anqing, China. ${ }^{3}$ Advanced Analytics Institute, Faculty of Engineering and IT, University of Technology Sydney, Broadway, NSW 2007, PO Box 123 Sydney, Australia.}

Received: 19 April 2018 Accepted: 9 July 2018

Published online: 03 August 2018

\section{References}

1. Anfinsen CB, Haber E, Sela M, Jr WF. The kinetics of formation of native ribonuclease during oxidation of the reduced polypeptide chain. Proc Natl Acad Sci U S A. 1961;47(9):1309-14.
2. Kabsch W, Sander C. Dictionary of protein secondary structure: pattern recognition of hydrogen-bonded and geometrical features. Biopolymers. 1983;22(12):2577-637.

3. Schmidler SC, Liu JS, Brutlag LD. Bayesian segmentation of protein secondary structure. J Comput Biol A J Comput Mol Cell Biol. 2000;7(1-2): 233-48.

4. Chu W, Ghahramani Z, Wild DL. A graphical model for protein secondary structure prediction. In: Proceedings 21st Annual International Conference on Machine Learning(ICML). New York: ACM; 2004. p. 161-8.

5. Hua S, Sun Z. A novel method of protein secondary structure prediction with high segment overlap measure: support vector machine approach. J Mol Biol. 2001;308(2):397-407.

6. Guo J, Chen H, Sun Z, Lin Y. A novel method for protein secondary structure prediction using dual-layer svm and profiles. Protein Struct Funct Bioinform. 2004;54(4):738-43.

7. Asai K, Hayamizu S, Handa K. Prediction of protein secondary structure by the hidden markov model. Bioinformatics. 1993;9(2):141.

8. Aydin Z, Altunbasak Y, Borodovsky M. Protein secondary structure prediction for a single-sequence using hidden semi-markov models. BMC Bioinformatics. 2006;7(1):178

9. Qian N, Sejnowski TJ. Predicting the secondary structure of globular proteins using neural network models. J Mol Biol. 1988;202(4):865-84

10. Jones DT. Protein secondary structure prediction based on position-specific scoring matrices. J Mol Biol. 1999;292(2):195.

11. Buchan DW, Minneci F, Nugent TC, Bryson K, Jones DT. Scalable web services for the psipred protein analysis workbench. Nucleic Acids Res. 2013;41(Web Server issue):349-57.

12. Faraggi $\mathrm{E}, \mathrm{Al} \mathrm{E}$. Spine $\mathrm{x}$ : improving protein secondary structure prediction by multistep learning coupled with prediction of solvent accessible surface area and backbone torsion angles. J Comput Chem. 2012;33(3): 259-67.

13. Baldi P, Brunak Sfrasconi P, Soda G, Pollastri G. Exploiting the past and the future in protein secondary structure prediction. Bioinformatics. 1999;15(11):937-46.

14. Chen J, Chaudhari NS. Cascaded bidirectional recurrent neural networks for protein secondary structure prediction. IEEE/ACM Trans Comput Biol Bioinforma. 2007:4(4):572-82.

15. Mirabello C, Pollastri G. Porter, paleale 4.0: high-accuracy prediction of protein secondary structure and relative solvent accessibility. Bioinformatics. 2013;29(16):2056-8.

16. Torrisi M, Kaleel M, Pollastri G. Porter 5: state-of-the-art ab initio prediction of protein secondary structure in 3 and 8 classes. 2018, https://www.biorxiv.org/content/early/2018/03/30/289033.

17. Zhou J, Troyanskaya OG. Deep supervised and convolutional generative stochastic network for protein secondary structure prediction. In: Proceedings of the 31st International Converenfe on Machine Learning (ICML). Bejing: PMLR; 2014. p. 745-53.

18. S $\phi$ nderby SK, Winther O. Protein secondary structure prediction with long short term memory networks. ArXiv e-prints. 2014; http://adsabs. harvard.edu/abs/2014arXiv1412.7828K.

19. Heffernan R, Yang Y, Paliwal K, Zhou Y. Capturing non-local interactions by long short-term memory bidirectional recurrent neural networks for improving prediction of protein secondary structure, backbone angles, contact numbers and solvent accessibility. Bioinformatics. 2017;33(18): 2842-9.

20. Wang S, Peng J, Ma J, Xu J. Protein secondary structure prediction using deep convolutional neural fields. Sci Rep. 2016;6.

21. Li Z, Yu Y. Protein secondary structure prediction using cascaded convolutional and recurrent neural networks. In: Proceedings of the 25th International Joint Conference on Artificial Intelligence (IJCAI). California: AAAI Press; 2016. p. 2560-7.

22. Busia A, Jaitly N. Next-step conditioned deep convolutional neural networks improve protein secondary structure prediction. In: conference on Intelligent Systems for Molecular Biology \& European Conference on Computational Biology (ISMB/ECCB 2017). Leesburg: International Society of Computational Biology; 2017.

23. Fang C, Shang Y, Xu D. Mufold-ss:new deep inception-inside-inception networks for protein secondary structure prediction. Protein Struct Funct Bioinforma. 2018;86(5):592-8.

24. Szegedy C, Vanhoucke V, loffe S, Shlens J, Wojna Z. Rethinking the Inception Architecture for Computer Vision. In: 2016 IEEE Conference on 
Computer Vision and Pattern Recognition (CVPR). Los Alamitos: IEEE Computer Society; 2016. p. 2818-26.

25. He K, Zhang X, Ren S, Sun J. Deep residual learning for image recognition. In: 2016 IEEE Conference on Computer Vision and Pattern Recognition (CVPR). Los Alamitos: IEEE Computer Society; 2016. p. 770-8.

26. Cho K, Merrienboer BV, Gulcehre C, Bahdanau D, Bougares F, Schwenk H, Bengio Y. Learning Phrase Representations Using RNN Encoder-Decoder for Statistical Machine Translation. In: Conference on Empirical Methods in Natural Language Processing. PA: Association for Computational Linguistics; 2014. p. 1724-34.

27. Guoli Wang RLDJ. Pisces: a protein sequence culling server. Bioinformatics. 2003;19(12):1589-91.

28. Li W, Godzik A. Cd-hit: a fast program for clustering and comparing large sets of protein or nucleotide sequences. Bioinformatics. 2006;22(13):1658

29. Altschul SF, Gertz EM, Agarwala R, Schaäffer AA, Yu YK. Psi-blast pseudocounts and the minimum description length principle. Nucleic Acids Res. 2009;37(3):815-24.

30. Jens M, Michael M, Anita Z, Felix S. Generation and evaluation of dimension-reduced amino acid parameter representations by artificial neural networks. J Mol Model. 2001;7(9):360-9.

31. Heffernan R, Paliwal K, Lyons J, Dehzangi A, Sharma A, Wang J, Sattar A, Yang $Y$, Zhou Y. Improving prediction of secondary structure, local backbone angles, and solvent accessible surface area of proteins by iterative deep learning. Sci Rep. 2015;5(11476).

32. Quan L, Lv Q, Zhang Y. Strum: structure-based prediction of protein stability changes upon single-point mutation. Bioinformatics. 2016;32(19): 2936.

33. Altschul SF, Madden TL, Schaäffer AA, Zhang J, Zhang Z, Miller W, Lipman DJ. Gapped blast and psi-blast: a new generation of protein database search programs. Nucleic Acids Res. 1997;25(17):3389-402.

34. Ordóñez FJ, Roggen D. Deep convolutional and Istm recurrent neural networks for multimodal wearable activity recognition. Sensors. 2016;16(1):115.

35. Schuster M, Paliwal KK. Bidirectional recurrent neural networks. IEEE Trans Signal Process. 1997;45(11):2673-681.

36. Jozefowicz R, Zaremba W, Sutskever I. An empirical exploration of recurrent network architectures. In: Proceedings of the 32nd International Converenfe on Machine Learning (ICML). New York: ACM; 2015. p. 171-80.

37. Hochreiter S, Schmidhuber J. Long short-term memory. Neural Comput. 1997;9(8):1735.

38. Lewis DD, Yang Y, Rose TG, Li F. Rcv1: A new benchmark collection for text categorization research. J Mach Learn Res. 2004;5(2):361-97.

39. Polikar R. Ensemble based systems in decision making. IEEE Circ Syst Mag. 2006;6(3):21-45.

40. Zemla A, Venclovas C, Fidelis K, Rost B. A modified definition of sov, a segment-based measure for protein secondary structure prediction assessment. Protein Struct Funct Bioinform. 1999;34(2):220-3.

41. Liu T, Wang Z. Sov_refine: A further refined definition of segment overlap score and its significance for protein structure similarity. Source Code Biol Med. 2018;13(1):1

Ready to submit your research? Choose BMC and benefit from:

- fast, convenient online submission

- thorough peer review by experienced researchers in your field

- rapid publication on acceptance

- support for research data, including large and complex data types

- gold Open Access which fosters wider collaboration and increased citations

- maximum visibility for your research: over $100 \mathrm{M}$ website views per year

At BMC, research is always in progress.

Learn more biomedcentral.com/submissions 